\title{
Studies of jet cross-sections and production properties with the ATLAS and CMS detectors
}

\author{
Nuno Anjos ${ }^{1, a, b}$ on behalf of the ATLAS and CMS Collaborations \\ ${ }^{1}$ Institut de Física d'Altes Energies (IFAE), The Barcelona Institute of Science and Technology, Campus \\ UAB, 08193 Bellaterra (Barcelona) Spain.
}

\begin{abstract}
Several characteristics of jet production in pp collisions have been measured by the ATLAS and CMS collaborations at the LHC. Measurements of event shapes and multi-jet production probe the dynamics of QCD in the soft regime and can constrain parton shower and hadronisation models. Measurements of multi-jet systems with a veto on additional jets probe QCD radiation effects. Double-differential cross-sections for threeand four-jet final states are measured at different centre-of-mass energies of pp collisions and are compared to expectations based on NLO QCD calculations. The distribution of the jet charge has been measured in di-jet events and compared to predictions from different hadronisation models and tunes. Jet-jet energy correlations are sensitive to the strong coupling constant. These measurements constitute precision tests of QCD in a new energy regime.
\end{abstract}

\section{Introduction}

Jets are narrow collimated clusters of stable particles (mainly hadrons) produced in high-energy particle interactions. In the theory of strong interactions, quantum chromodynamics (QCD), jets arise from the fragmentation of partons (quarks and gluons). In a first (perturbative) phase, partons with high-transverse momentum $\left(p_{\mathrm{T}}\right)$ are produced in a hard-scattering process. Partons evolve from hard to soft scales by a process of emission of additional gluons and production of quark-antiquark pairs, known as the parton shower (PS). This phase can be described by perturbative QCD (pQCD) approximations. In the second (non-perturbative) phase, jet evolution is an interplay between the hadronisation process and the underlying event (UE). The hadronisation process governs the transition from partons to hadrons. The UE represents initial-state radiation, multiple parton interactions and colourreconnection effects. Non-perturbative (NP) effects are most pronounced at low $p_{\mathrm{T}}$, and are accounted for through the use of phenomenological models that are tuned to the data.

ATLAS [1] and CMS [2] are multi-purpose experiments at the Large Hadron Collider (LHC), the proton-proton (pp) collider at CERN. This paper presents several studies [3-11] of jet production

\footnotetext{
a e-mail: nuno.anjos@cern.ch

${ }^{b}$ Work supported by the Beatriu de Pinós program managed by Agència de Gestió d'Ajuts Universitaris i de Recerca with the support of the Secretaria d'Universitats i Recerca of the Departament d'Economia i Coneixement of the Generalitat de Catalunya, and the Cofund program of the Marie Curie Actions of the 7th R\&D Framework Program of the European Union. Work partially supported by MINECO under grants SEV-2012-0234, FPA2013-48308, and FPA2012-38713, which include FEDER funds from the European Union.
} 
performed by ATLAS and CMS with data taken at centre-of-mass energies $(\sqrt{s})$ of 7 and $8 \mathrm{TeV}$ during Run 1 of the LHC, from 2010 until 2012. The ATLAS and CMS detectors cover a solid angle of almost $4 \pi$. Closest to the interaction point are precise tracking devices, surrounded by noncompensating calorimeters and muon chambers. All analyses presented here use single jet calorimeter based triggers to select events online. The ATLAS analysis in Sec. 3 also uses minimum bias triggers. As inputs to offline jet reconstruction, ATLAS uses topoclusters [12] while CMS uses Particle Flow (PF) objects [13]. The topoclustering algorithm groups cells from the finely segmented calorimeters in three-dimensional topological clusters according to an iterative procedure designed to suppress noise. The PF algorithm utilizes the best energy measurements of each particle candidate from the most suitable combination of detector components. Both experiments use the anti- $k_{T}$ [14] algorithm as implemented in the FASTJET [15] package to reconstruct jets. The values of the jet distance parameter $R$ used by ATLAS are 0.4 and 0.6 , while CMS uses 0.5 and 0.7 . Both experiments apply a factorized jet calibration procedure with corrections for pile-up and jet energy scale.

Both experiments apply unfolding procedures to correct data for detector effects and allow comparisons with theory. Theoretical predictions are obtained from Monte Carlo (MC) event generators. The Pythia 6 [16], Pythia 8 [17], Herwig 6 [18] and Herwig++ [19] MCs complement leadingorder (LO) $2 \rightarrow 2$ matrix elements (ME) with parton showers to simulate higher-order processes. MADGRAPH [20] supplies an improved LO ME calculation with $2 \rightarrow 4$ outgoing partons that can be matched to one of the above PS MCs. AlPGEN provides LO MEs for $2 \rightarrow n$ multi-parton final states, with $n \leq 6$, interfaced with Herwig 6 for the PS. The Powheg framework [21-23] provides a full next-to-leading-order (NLO) di-jet calculation accounting for the emission of an additional third hard parton, and can also be matched to a PS MC.

\section{Hadronic Event Shapes}

Event shapes (ES) are observables that measure the energy flow in an event. They are combinations of the momenta of final state particles in a number related to the event geometry. In terms of hadronic jet production, ES are an indirect probe of multi-jet topologies. They are theoretically defined in an infrared- and collinear-safe manner and can be computed in pQCD. A large set of ES sensitive to QCD dynamics from soft (hadronisation) to hard (multi-jet radiation) scales has been proposed [24, 25] for studies in pp collisions. CMS has studied ES [3] using a dataset with an integrated luminosity of $5 \mathrm{fb}^{-1}$ recorded at $\sqrt{s}=7 \mathrm{TeV}$ during 2011. The ES studied by CMS are the transverse thrust $\left(\tau_{\perp}\right)$, jet broadening $\left(B_{\text {tot }}\right)$, jet mass (both total, $\rho_{\text {tot }}$, and in the transverse plane, $\left.\rho_{\text {tot }}^{\mathrm{T}}\right)$ and the third-jet resolution parameter $\left(\mathrm{T}_{23}\right)$. Events are selected with at least one $R=0.5$ jet with transverse momentum $p_{\mathrm{T}}>110 \mathrm{GeV}$ and absolute pseudorapidity $|\eta|<2.4$. The ES distributions are studied in five leading jet $p_{\mathrm{T}}$ ranges and compared to predictions from standard and improved LO MCs, combined with several PS and UE tunes: D6T [26], Perugia-P0 [27], Z2 [28] and tune4C [29]. Fig. 1 (left) shows the $\tau_{\perp}$ distribution for one range of leading jet $p_{\mathrm{T}}$. The MCs agrees with data to within $20 \%$. ES more sensitive to the longitudinal energy flow or hard parton emission, such as $\rho_{\text {tot }}$ in Fig. 1 (right), exhibit larger disagreement between data and MC. MADGRAPH consistently reproduces all distributions within uncertainties.

\section{Di-jet Azimuthal Decorrelation}

At leading order (LO) in pQCD, two final-state partons balanced in $p_{\mathrm{T}}$ are produced in a back-to-back topology. The azimuthal angular separation between the two $p_{\mathrm{T}}$ leading jets, $\Delta \phi=\left|\phi_{1}-\phi_{2}\right|$, equals $\pi$. The production of a third high- $p_{\mathrm{T}}$ jet, however, leads to a decorrelation in azimuthal angle. The smallest achievable value of $\Delta \phi=2 \pi / 3$ is reached with a symmetric star-shaped three-jet configuration. 

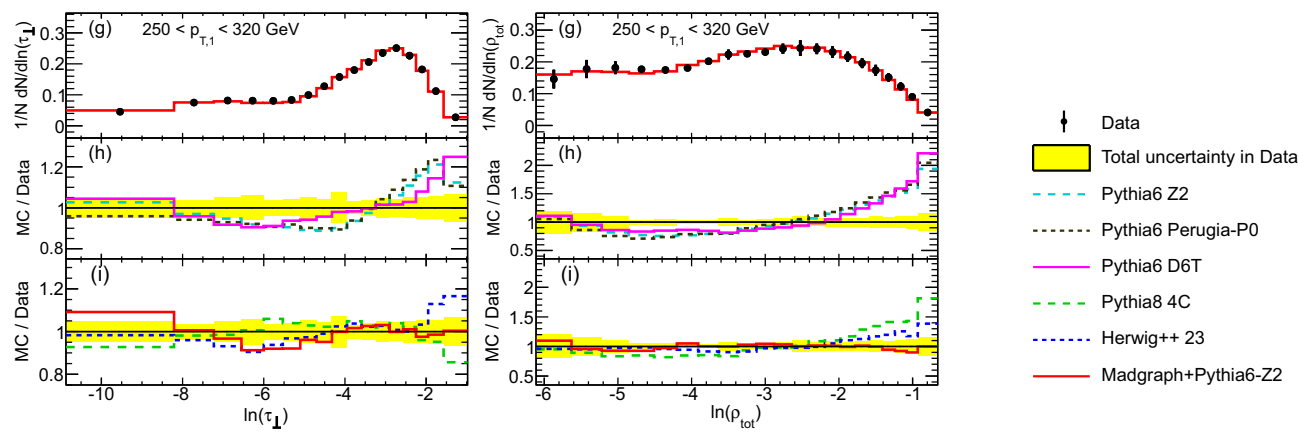

Figure 1. The transverse thrust (left) and jet mass (right) distributions in the 250 to $320 \mathrm{GeV}$ leading jet $p_{\mathrm{T}}$ range from $5 \mathrm{fb}^{-1}$ of CMS data recorded at $\sqrt{s}=7 \mathrm{TeV}$, compared to MADGraph predictions. The prediction/data ratios for the MC models listed in the caption are also shown [3].

If more than three jets are found, $\Delta \phi$ decreases and approaches the limit of the jet size. Therefore, the measurement of the di-jet azimuthal decorrelation is a way to study multi-jet production and test NLO predictions without actually measuring jets beyond the leading two. CMS measured the di-jet azimuthal decorrelation [4] using $19.7 \mathrm{fb}^{-1}$ of data recorded at $\sqrt{s}=8 \mathrm{TeV}$ in 2012. Events with at least two $R=0.7$ jets with rapidity $|y|<2.5$ and (sub)leading jet $p_{\mathrm{T}}>(100) 200 \mathrm{GeV}$ are selected. To reduce the background from $t \bar{t}$ and heavy vector boson production to hard-QCD jet events, events for which the ratio of missing transverse momentum $E_{\mathrm{T}}^{\text {miss }}$ to total transverse energy $\sum E_{\mathrm{T}}$ is larger than 0.1 are rejected. The di-jet cross-section is measured as a function of $\Delta \phi$ and normalized to the total di-jet cross-section, reducing many experimental and theoretical uncertainties. The measured differential cross-section is shown in Fig. 2 (left) in slices of the leading jet $p_{\mathrm{T}}$ up to $2.2 \mathrm{TeV}$. It is compared with LO and NLO MC predictions. MADGRAPH agrees with data throughout the entire $\Delta \phi$ range, Pythia 8 also provides a reasonable description of the data, while the other MCs overestimate the data in specific regions.

Although fixed order computations provide a good description of jet production, for certain observables and in some regions of the phase space higher-order contributions are important and must be resummed to all orders. This is the case for di-jet events with large rapidity separation between the two jets, $\Delta y$, and/or when a veto is applied on additional jets with $p_{\mathrm{T}}>Q_{0}$ produced in the rapidity interval between them; $Q_{0}$ is called a veto scale. There are two available resummation approaches, BFKL and DGLAP. BFKL resums the higher orders in terms of $\ln (1 / x)$ while DGLAP does it in terms of $\ln \left(Q^{2}\right)$. The DGLAP inspired procedure is implemented in PowHEg interfaced with Herwig 6 or Pythia 8. The predictions based on BFKL are generated with HeJ [30, 31], alone or interfaced with Ariadne [32]. Hej resums leading-logarithms relevant in the Mueller-Navelet limit. Ariadne provides parton showers based on the colour-dipole cascade model. ATLAS has studied jet production in events with large- $y$ separated di-jets and a veto on a third in-between jet [5], using $36.1 \mathrm{pb}^{-1}$ and $4.5 \mathrm{fb}^{-1} \mathrm{of}^{-}$ data collected at $\sqrt{s}=7 \mathrm{TeV}$ during 2010 and 2011 respectively. Events with at least two $R=0.6$ jets, with (sub)leading jet $p_{\mathrm{T}}>(50) 60 \mathrm{GeV}$ are selected. For 2010 data, the full rapidity range of $|y|<4.9$ was used. For 2011 data, $|\Delta y| \leq 1$ and $|y|<2.4$ are required on the two leading jets. Events are classified into two categories: the inclusive and gap di-jet events. An event contributes to the gap sample if there is no jet with $p_{\mathrm{T}}>Q_{0}$ in the rapidity interval between the two leading jets. The veto scale $Q_{0}$ was set to $p_{\mathrm{T}}>20$ (30) GeV for data collected in 2010 (2011). The gap fraction, the ratio of the di-jet cross-sections measured using the gap and the inclusive samples, and the mean multiplicity 

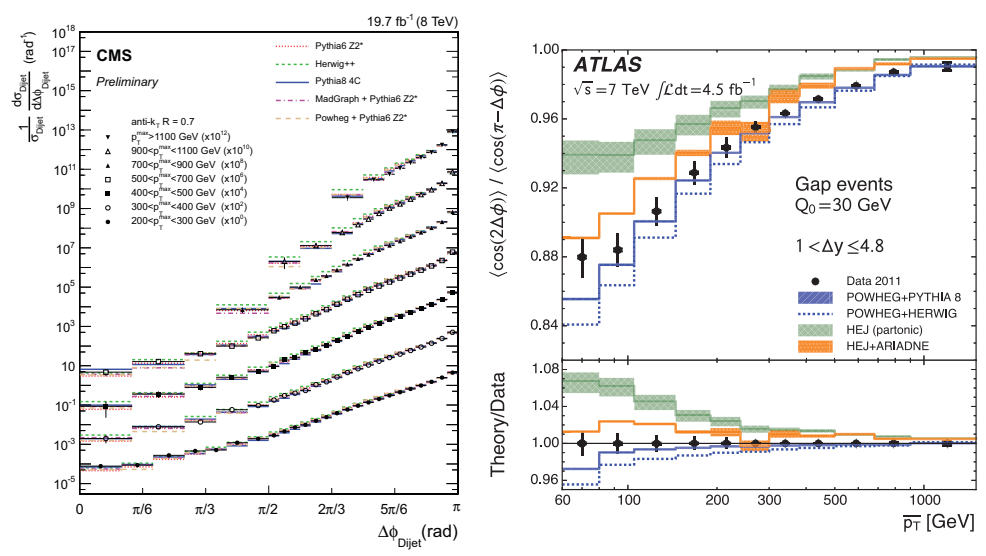

Figure 2. Normalised CMS di-jet cross-section, differential in $\Delta \phi$ for seven leading $p_{\mathrm{T}}$ regions, compared to $\mathrm{MC}$ predictions and scaled for presentation purposes (left) [4]. Ratio of the first two angular moments measured in the ATLAS di-jet gap sample, compared to MC predictions (right) [5].

of jets with $p_{\mathrm{T}}>Q_{0}$ in the gap are measured. Powheg+Pythia 8 and HeJ+Ariadne provide the best agreement with data. The observable with best discrimination is the ratio of the first two angular moments, $\langle\cos (2 \Delta \phi)\rangle /\langle\cos (\pi-\Delta \phi)\rangle$. It is shown in Fig. 2 (right) for the gap sample. The data is below the Hej+Ariadne prediction and slightly above the Powheg+Pythia 8 prediction.

\section{Multi-jet Topologies}

Multi-jet topologies test both higher order effects in pQCD and NP dynamics. Topological variables are sensitive to QCD colour factors, gluon spin structure and hadronisation.

Both ATLAS and CMS have measured the double differential tri-jet cross-section with data recorded at $\sqrt{s}=7 \mathrm{TeV}$ in 2011. The cross-sections are presented as a function of the three-jet mass and in bins of an angular variable. The CMS measurement [6] uses a data set of $5 \mathrm{fb}^{-1}$. Events where at least three $R=0.7$ jets are found with $|y| \leq 3$ and $p_{\mathrm{T}}>100 \mathrm{GeV}$ are selected. The angular variable is the maximum jet rapidity, $|y|_{\max }=\max \left(\left|y_{1}\right|,\left|y_{2}\right|,\left|y_{3}\right|\right)$. Two bins of $|y|_{\max }$ are defined, $|y|_{\max } \leq 1$ and $1<|y|_{\max } \leq 2$, with events outside this range being discarded. The ATLAS measurement [7] uses a data set of $4.5 \mathrm{fb}^{-1}$. Events where at least three jets are found with $|y| \leq 3$ and with $p_{\mathrm{T}}>150,100$ and $50 \mathrm{GeV}$, respectively, are selected. The angular variable is the sum of the jet separations, $\left|Y^{*}\right|=\left|\Delta y_{1,2}\right|+\left|\Delta y_{2,3}\right|+\left|\Delta y_{3,4}\right|$, and five bins of $\left|Y^{*}\right|$ were defined. Fig. 3 shows the measured tri-jet cross-section as a function of the three-jet mass in bins of the angular variable for CMS (left) and ATLAS (right). The ATLAS results on display use jets with $R=0.4$, but results were also obtained for $R=0.6$. Also shown is the comparison with the NLOJET++ [33] prediction with the CT10 [34] parton distribution function (PDF) set and corrected for NP effects. Good agreement between the data and the theoretical predictions was found by both experiments.

ATLAS measured four-jet differential cross-setions [8] using $20.3 \mathrm{fb}^{-1}$ of data recorded at $\sqrt{s}=$ $8 \mathrm{TeV}$ in 2012. Events with at least four $R=0.4$ jets within $|y|<2.8$, leading jet $p_{\mathrm{T}}>100 \mathrm{GeV}$, three subleading jets with $p_{\mathrm{T}}>64 \mathrm{GeV}$ and minimal angular distance between any pair of four leading jets larger than 0.65 are selected. Cross-sections are measured as a function of several variables including the four-jet mass, as shown in Fig. 4 (left). Results are compared to predictions from several LO and NLO MCs.

CMS studied distributions of topological observables in inclusive three- and four-jet events [9] using $5.1 \mathrm{fb}^{-1}$ of data recorded at $\sqrt{s}=7 \mathrm{TeV}$ in 2011. All $R=0.5$ jets are required to have $p_{\mathrm{T}}>50 \mathrm{GeV}$ and $|y|<2.5$. The leading jet $p_{\mathrm{T}}$ must be in the $190-300 \mathrm{GeV}$ range or above $500 \mathrm{GeV}$ 

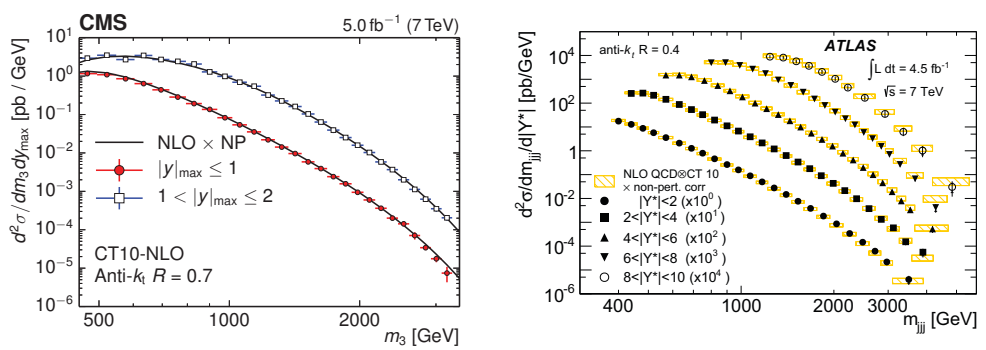

Figure 3. The tri-jet cross-section as a function of the three-jet mass $m_{3}=m_{j j j}=\sqrt{p_{1}^{2}+p_{2}^{2}+p_{3}^{2}}$ and in bins of the angular variable, measured by CMS (left) [6] and ATLAS (right) [7] compared with NLO predictions corrected for NP effects.
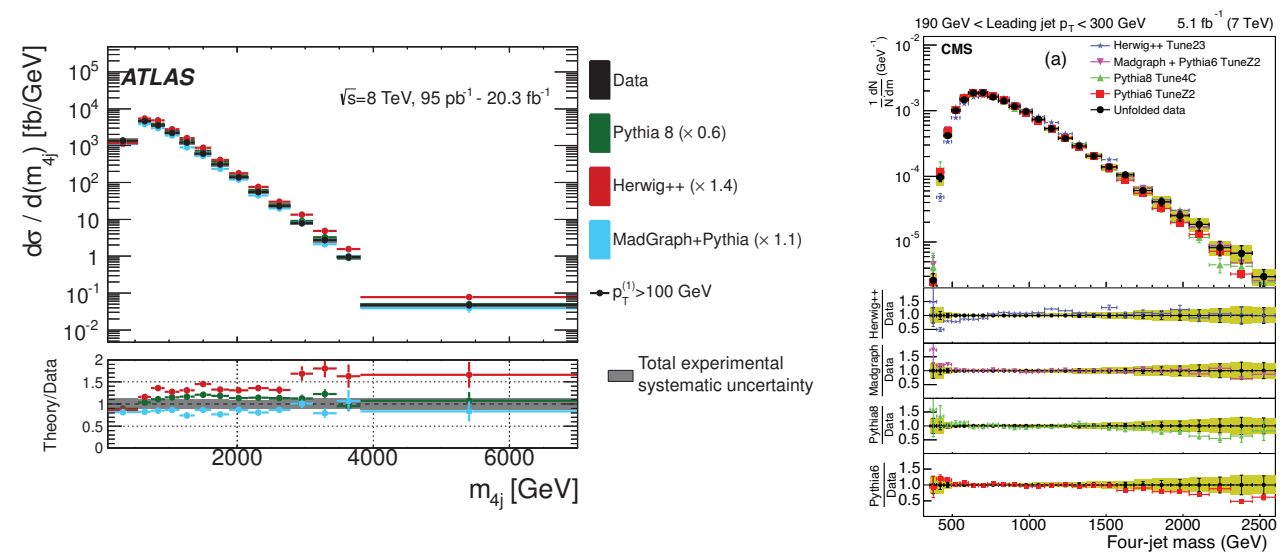

Figure 4. Four-jet differential cross-section a function of $m_{4 j}=\sqrt{p_{1}^{2}+p_{2}^{2}+p_{3}^{2}+p_{4}^{2}}$ measured by ATLAS at $\sqrt{s}=8 \mathrm{TeV}$ (left) [8]. Distribution of $m_{4 j}$ in events with leading jet in the 190-300 GeV range measured by CMS at $\sqrt{s}=7 \mathrm{TeV}$ (right) [9]. Both are compared to different theoretical predictions.

to satisfy trigger criteria. Events with at least three (four) jets satisfying the selection are classified as three(four)-jet events. Distributions are obtained as a function of multiple variables, including dedicated angular variables and the three- and four-jet masses, the last one shown in Fig. 4 (right). Results are compared to predictions from several LO MCs.

Both ATLAS and CMS find good overall agreement between data and theory, with discrepancies in specific regions. The LO MCs can disagree with the data due to the missing higher multiplicities, while the NLO MCs can have large uncertainties, specially at low momenta.

\section{Jet Charge}

Due the confining nature of the strong force, the electric charge of quarks is not directly observable. It is however possible to define a jet charge observable as the momentum-weighted sum of the charges of tracks associated to a jet. This observable is sensitive to the charge of the initiating quark or gluon and can provide constraints on models of jet formation. ATLAS measured the jet charge [10] using $20.3 \mathrm{fb}^{-1}$ of data recorded at $\sqrt{s}=8 \mathrm{TeV}$ in 2012. Events are required to have at least two $R=0.4$ jets 

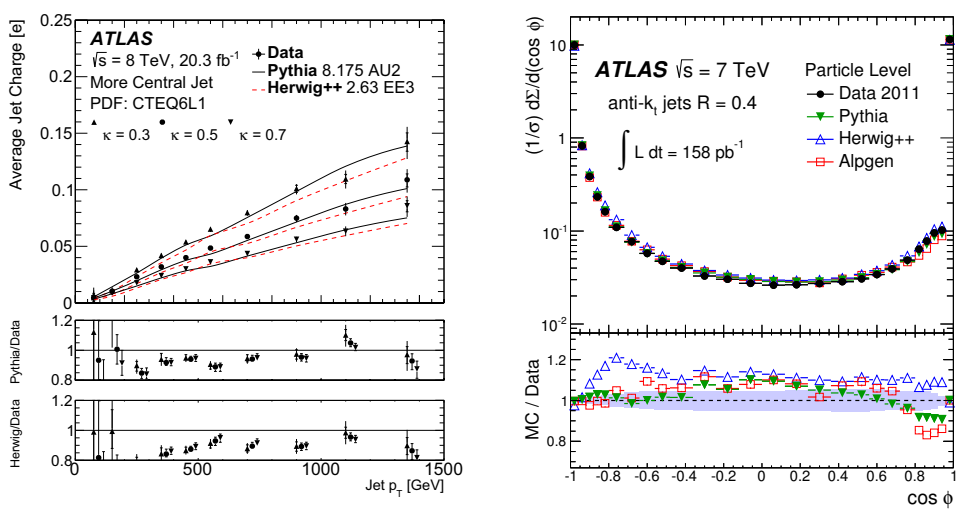

Figure 5. The average jet charge in units of positron charge measured by ATLAS as a function of jet $p_{\mathrm{T}}$, for $\kappa=0.3,0.5,0.7$ and for the most central jets, using the CT10 PDF set. (left) [10]. The TEEC distribution measured by ATLAS data with comparisons to $\mathrm{MC}$ expectations.(right) [11].

with $p_{\mathrm{T}}>50 \mathrm{GeV}$ in the central region, $(|y|<2.1)$, and only the two leading jets are considered for the jet charge measurement. To select di-jet topologies, the two leading jets must have $p_{\mathrm{T}, 1} / p_{\mathrm{T}, 2}<1.5$. The jet with the smallest (largest) $|\eta|$ is classified as the most central (most forward) jet. The jet charge $Q_{J}$ of a jet $J$ is calculated using a transverse-momentum weighting, $Q_{J}=\left(1 / p_{\mathrm{T}, J}^{\kappa}\right) \sum_{i} q_{i} p_{\mathrm{T}, i}^{\kappa}$, where $i$ runs over all tracks associated to jet $J, p_{\mathrm{T}, i}$ is the transverse momentum of track $i$ with associated charge $q_{i}, p_{\mathrm{T}, J}$ is the transverse momentum of the jet and $\kappa$ is a free regularisation parameter (values of $0.3,0.5$ and 0.7 are used) that controls the sensitivity to soft radiation. The average of the jet charge distribution is shown in Fig. 5 (left) compared to several MCs. The calculations are generally about $10 \%$ below the data, possibly due to fragmentation modeling. The jet charge increases with jet $p_{\mathrm{T}}$, which is expected to happen due to the increase in up-flavour jets.

\section{Transverse Energy-energy Correlations}

The transverse energy-energy correlation (TEEC) [35] is an event shape proposed for the study of hadronic collisions. that exhibits a quadratic dependence on the strong coupling constant. The TEEC distribution is obtained by calculating the cosines of the angles in the transverse plane between all possible pairs of jets in each event, $\phi_{i j}$. Every pair $(i, j)$ represents an entry in the distribution, which is then weighted with the normalised product of the transverse energies. ATLAS measured the TEEC [11] using $158 \mathrm{pb}^{-1}$ of data recorded at $\sqrt{s}=7 \mathrm{TeV}$ in 2011. The event selection requires at least two $R=0.4$ jets with $p_{\mathrm{T}}>50 \mathrm{GeV}$ and $|\eta|<2.5$, as well as $p_{\mathrm{T}, 1}+p_{\mathrm{T}, 2}>500 \mathrm{GeV}$. The comparison of the measured TEEC spectrum to the MC predictions is shown in Fig. 5 (right). The description of the TEEC is good in the back-to-back region $\cos \phi \simeq-1$ for both PYтHIA 6 and Alpgen+Herwig. Differences up to $10 \%$ are observed in the central part, while the region of small angles shows differences as large as about $15 \%$. The description by HeRwIG++ is poorer. The $\alpha_{s}\left(m_{\mathrm{Z}}^{2}\right)$ value is extracted by performing a $\chi^{2}$ fit of theoretical predictions to the TEEC data. The theoretical predictions are provided by NLO pQCD with NP corrections. This yields $\alpha_{s}=0.1173 \pm 0.0010$ (exp.) ${ }_{-0.0020}^{+0.0063}$ (scale) \pm 0.0017 (PDF) \pm 0.0002 (NP corrections), where the uncertainties are experimental, from the scale choice, the choice of PDFs and the NP corrections, respectively.

\section{Conclusions}

Multi-jet production in pp collisions was extensively studied by the ATLAS and CMS collaborations at centre-of-mass energies of 7 and $8 \mathrm{TeV}$ during Run 1 of the LHC . Studies presented include hadronic 
event shapes, TEEC, jet charge in di-jet events, di-jet azimuthal decorrelation with or without a jet veto, double-differential cross-sections for three- and four-jet final states, and multi-jet topological distributions. Measurements were compared with NLO predictions corrected for NP effects, and ME generators at LO and NLO. Overall, good agreement between data and theory was found, and the TEEC were used to measure the strong coupling constant.

\section{References}

[1] ATLAS Collaboration, JINST 3, S08003 (2008)

[2] CMS Collaboration, JINST 3, S08004 (2008)

[3] CMS Collaboration, JHEP 10, 087 (2014).

[4] CMS Collaboration, CMS-PAS-SMP-14-015, https://cdsweb.cern.ch/record/2019401.

[5] ATLAS Collaboration, Eur. Phys. J. C 74, 3117 (2014).

[6] CMS Collaboration, Eur. Phys. J. C 75, 186 (2015).

[7] ATLAS Collaboration, Eur. Phys. J. C 75, 228 (2015).

[8] ATLAS Collaboration, arXiv:1509.07335 [hep-ex].

[9] CMS Collaboration, Eur. Phys. J. C 75, 302 (2015).

[10] ATLAS Collaboration, arXiv:1509.05190 [hep-ex].

[11] ATLAS Collaboration, Phys. Lett. B 750, 427 (2015).

[12] W. Lampl et al, ATL-LARG-PUB-2008-002, http://cdsweb.cern.ch/record/1099735.

[13] CMS Collaboration, CMS-PAS-PFT-09-001, https://cdsweb.cern.ch/record/1194487.

[14] M. Cacciari, G. P. Salam, and G. Soyez, JHEP 04063 (2008).

[15] M. Cacciari, G. P. Salam, and G. Soyez, Eur. Phys. J. C 72, 1896 (2012).

[16] T. Sjöstrand, S. Mrenna and P. Z. Skands, JHEP 05, 026 (2006).

[17] T. Sjöstrand, S. Mrenna and P. Z. Skands, Comput. Phys. Commun. 178, 852 (2008).

[18] G. Corcella et al., JHEP 0101, 010 (2001).

[19] M. Bähr et al., Eur. Phys. J. C 58, 639 (2008).

[20] J. Alwall et al., JHEP 06, 128 (2011).

[21] S. Frixione, P. Nason, and C. Oleari, JHEP 11, 070 (2007).

[22] S. Alioli, P. Nason, C. Oleari, and E. Re, JHEP 06 043, (2010).

[23] P. Nason, JHEP 0411, 040 (2004).

[24] A. Banfi, G. P. Salam and G. Zanderighi, JHEP 08, 062 (2004).

[25] A. Banfi, G. P. Salam and G. Zanderighi, JHEP 06, 038 (2010).

[26] R. Field, Acta Phys. Polon. B 39, 2611 (2008).

[27] P. Z. Skands, Phys. Rev. D 82, 074018 (2010).

[28] R. Field, Acta Phys. Polon. B 42, 2631 (2011).

[29] R. Corke and T. Sjöstrand, JHEP 03, 032 (2011).

[30] J. R. Andersen and J. M. Smillie, Nucl.Phys.Proc.Suppl. 205-206, 205-210 (2010).

[31] J. R. Andersen and J. M. Smillie, JHEP 1106, 010 (2011).

[32] L. Lonnblad, Comput.Phys.Commun. 71, 15-31 (1992).

[33] Z. Nagy, Phys. Rev. D 68094002 (2003).

[34] H.-L. Lai et al., Phys. Rev. D 82, 074024 (2010).

[35] A. Ali, E. Pietarinen and W. J. Stirling, Phys. Lett. B 141, 447 (1984). 